\title{
Surgical management of obstructive sleep apnea
}

\author{
PUSHKAR MEHRA, BDS, DMD, AND LARRY M. WOLFORD, DMD
}

Obstructive sleep apnea (OSA) syndrome is a common disorder that has recently received much attention by the medical community due to its potentially serious physiological consequences. The clinical significance of OSA results from hypoxemia and sleep fragmentation caused by collapse of the airway, which leads to apnea or hypopnea during sleep. This paper reviews common surgical techniques used for clinical management of OSA patients, with emphasis on jaw advancement surgical procedures.

$\mathrm{O}$ bstructive sleep apnea (OSA) syndrome is a relatively common disorder that involves periodic partial or total collapse of the pharyngeal airway during sleep. This results in progressive asphyxia, which increasingly stimulates breathing efforts against the collapsed airway, typically until the patient is awakened from sleep (1).

Clinical sequelae of OSA result from the hypoxemia and sleep fragmentation (2). The respiratory disturbance index is the number of apneic or hypopneic events per hour during sleep, determined by polysomnography, and is used to quantify the severity of OSA. Additional symptoms include snoring, daytime sleepiness, and fatigue. As OSA progresses, cognitive dysfunction, inability to concentrate, memory and judgment impairment, irritability, and depression can develop, leading to work and social problems. Systemic consequences of OSA include hypertension, cardiac arrhythmias, pulmonary hypertension, cor pulmonale, left ventricular dysfunction, stroke, and death (2).

Nonsurgical options for treating OSA include weight loss, alteration of sleep posture, oral appliance therapy, external nasal support devices, pharmacological therapy, and continuous positive airway pressure (CPAP) therapy (1). Surgical treatment options include tracheostomy, mandibular osteotomy with genioglossus or inferior border advancement, uvulopalatopharyngoplasty (UPPP), laser-assisted uvuloplasty (LAUP), reduction glossectomy, internal and external nasal reconstruction, tonsillectomy and adenoidectomy, and advancement of the upper and lower jaws. We review the various surgical techniques and present some of our clinical and research experience in the management of patients with OSA syndrome.

\section{TRACHEOSTOMY}

Permanent tracheostomy was the first efficacious procedure and the most common procedure used for treatment of OSA in the 1970s and 1980s $(3,4)$. Tracheostomy has a very high success rate in reversing OSA symptoms, except possibly for patients

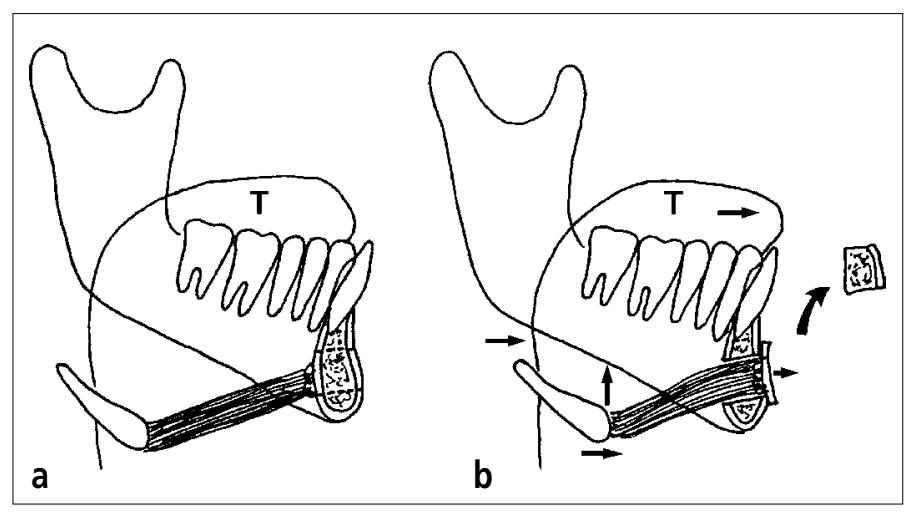

Figure 1. (a) The anterior mandibular osteotomy with genioglossus advancement procedure. A surgical access window is created in the anterior mandible by cutting through the outer and inner cortices and medullary bone. (b) The outer cortex and medullary bone have been removed. The inner cortex incorporating the genial tubercles (with attached musculature) has been anteriorly repositioned replacing the removed outer cortex. This simultaneously advances the hyoid bone and base of the tongue ( $\mathrm{T}$ ) (arrows).

with obesity-hypoventilation syndrome, as it bypasses all potential upper airway obstructive sites. Despite its effectiveness, tracheostomy is rarely used as first-line treatment of OSA because of serious disadvantages, which include tracheal stenosis, blood vessel erosion, recurrent purulent bronchitis, speech difficulties, and aesthetic disfigurement.

\section{ANTERIOR MANDIBULAR OSTEOTOMY WITH GENIOGLOSSUS ADVANCEMENT}

This procedure involves an osteotomy in the anterior mandible, creating a block segment that incorporates the genial tubercles and associated muscle attachments (Figure 1a). The bony block is then repositioned anteriorly to advance and suspend the hyoid bone to the mandible (Figure 1b). Riley et al reported a $67 \%$ success rate, with failures related to obesity and abnormal mandibular skeletal development (5). This procedure has the advantages of being offered on an outpatient basis and being relatively minimally invasive. Its disadvantages are that it does

From the Department of Oral and Maxillofacial Surgery, Baylor University Medical Center, Dallas, Texas. Dr. Mehra is now with the Department of Oral and Maxillofacial Surgery at Boston University School of Dental Medicine, Boston, Massachusetts.

Corresponding author: Larry M. Wolford, DMD, 3409 Worth Street, Suite 400, Dallas, Texas 75246. 


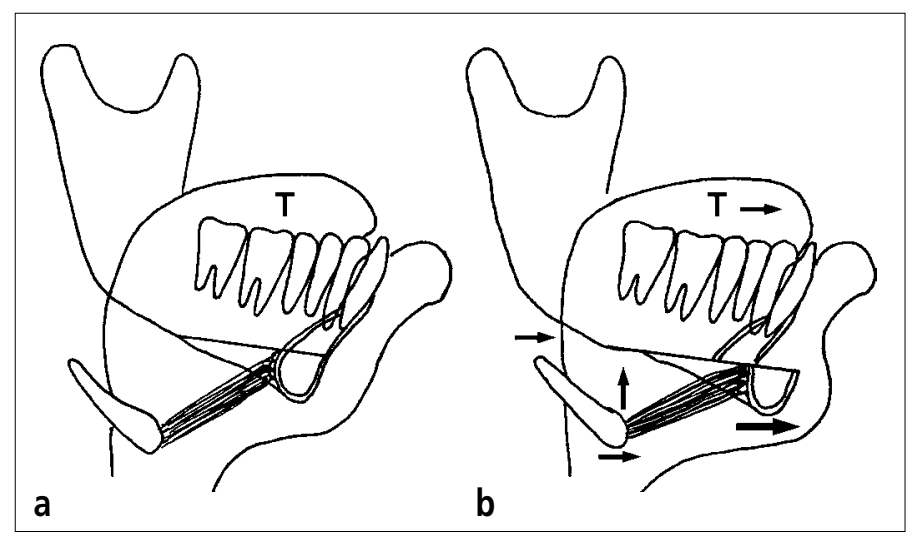

Figure 2. (a) The anterior mandibular horizontal osteotomy with genioglossus muscle and inferior border advancement (genioplasty). The osteotomy is made through both outer and inner cortices of the mandible. (b) The genioplasty segment has been advanced, causing a simultaneous advancement of the hyoid bone, base of the tongue ( $T$ ), and inferior border of the mandible (arrows).

not enlarge the oral cavity; genioglossus muscles can become detached, negating its effectiveness; and it is not effective in more severe OSA cases.

\section{ANTERIOR MANDIBULAR OSTEOTOMY WITH INFERIOR BORDER ADVANCEMENT (GENIOPLASTY)}

This procedure consists of an anterior mandibular horizontal osteotomy with genioglossus muscle and inferior border advancement (Figure 2). Advantages and disadvantages of this technique are similar to those of the Riley technique described above (5). However, this procedure results in a change in facial profile and may be beneficial for patients with aesthetic deficiency in the chin area.

\section{UVULOPALATOPHARYNGOPLASTY}

UPPP was first described by Ikematsu in 1964 for treatment of habitual snoring (6). Fujita et al modified the technique to increase the oropharyngeal airway space by excising the uvula and 8 to $15 \mathrm{~mm}$ of the posterior aspect of the soft palate, as well as the redundant lateral pharyngeal wall mucosa (7). Although UPPP has resulted in symptomatic improvement from habitual snoring in up to $90 \%$ of cases, only $41 \%$ to $66 \%$ of patients see improvement or elimination of OSA and results may worsen over time (8-11). The reason UPPP can fail is that the procedure addresses the obstruction at the soft palate area only, without improving the airway at the base of the tongue (hypopharyngeal area) or nasal cavity. In addition, scar contracture at the posterior border of the soft palate can create a "curtain" effect, pulling the soft palate downward against the tongue and causing significant transverse narrowing between the posterior faucial pillars, further contributing to OSA. Complications from UPPP include nasal regur- gitation, velopharyngeal incompetence, hypernasal speech, palatal stenosis, and residual OSA.

Wolford developed a modification of the traditional UPPP procedure that minimizes incisions along the posterior border of the soft palate, decreases postsurgical scarring, and predictably shortens the soft palate length, effectively improving the oropharyngeal airway space after surgery (Figure 3 ).

\section{LASER-ASSISTED UVULOPLASTY}

LAUP has gained popularity for treatment of snoring with reports of $80 \%$ to $85 \%$ success rates (4). Its advantages over UPPP are that it is an outpatient procedure under local anesthesia, it can be repeated, and it has decreased postoperative complications. However, a recent report on the efficacy of LAUP for treating OSA showed that $27 \%$ of the patients had a good response, $34 \%$ had a poor response, and 30\% got worse after surgery (12).

\section{REDUCTION GLOSSECTOMY}

True or relative macroglossia may be observed in some OSA patients $(4,13)$. An enlarged tongue can decrease the posterior airway space (PAS) at the oropharynx. Computerized axial tomography has confirmed that tongue volume increases with increasing obesity. However, the tongue may be of normal size but appear larger than normal if the volume of the oral cavity is decreased because of retropositioned jaws. If true macroglossia exists, a reduction glossectomy can be done, removing the anterior and a portion of the middle third of the tongue (Figure 4). Taste and sensation are minimally affected after surgery $(4,13)$. Wolford et al have previously presented the diagnosis, indications, techniques, and results of reduction glossectomy for treatment of macroglossia (13).

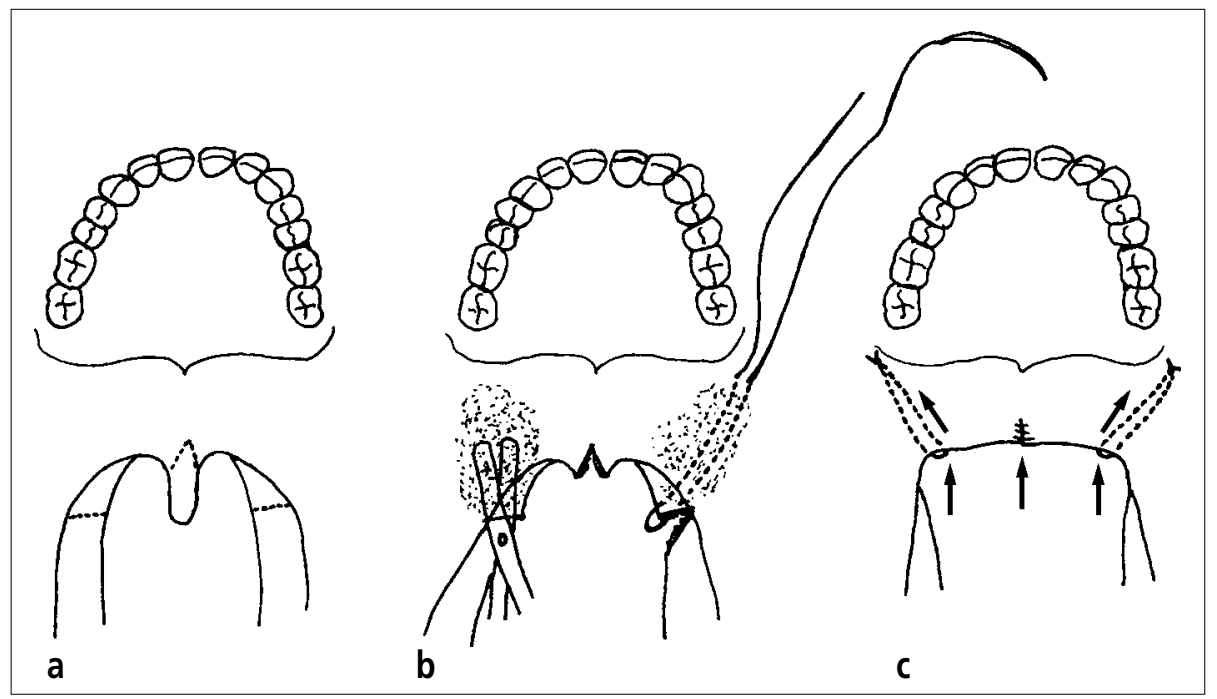

Figure 3. (a) Wolford's modified uvulopalatopharyngoplasty procedure. Note the hypertrophic uvula and the narrow transverse dimension between the right and left faucial pillars. The incisions are outlined at the base of the posterior faucial pillar bilaterally and at the base of the uvula. (b) Following completion of in cisions, the uvula flaps are closed. Dissection into the lateral aspect of the soft palatal tissues is performed in a submucosal plane between the oral and nasal sides with right-angled scissors. A mattress suture is passed submucosally from the base of the maxillary tuberosity to the dissected lateral flap of the posterior faucial pillar. The suture is passed through the end of the flap and tunneled back to the maxillary tuberosity area. The sutures are then tightened to shorten the soft palate to the desired dimension. (c) Postsurgical view of the velopharyngeal structures after a modified uvulopalatopharyngoplasty procedure. Note the increased transverse dimension of the oropharyngeal airway after lateralization and forward positioning of the posterior faucial pillars. 


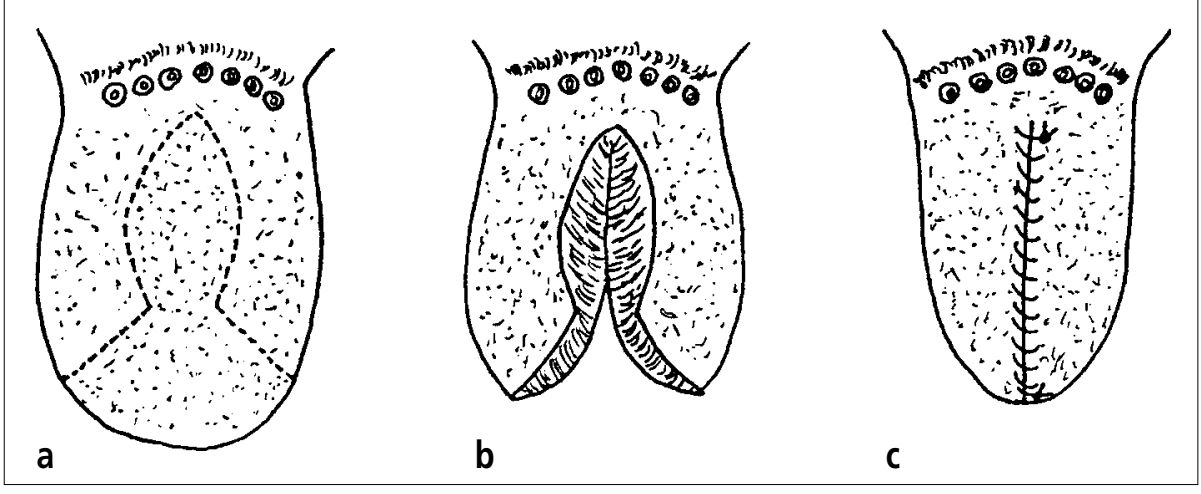

Figure 4. (a) The "keyhole" technique is used for surgical reduction of tongue size. Markings show the planned incisions. (b) The anterior middle part of the tongue has been resected and removed. (c) Suturing of the tongue reestablishes anatomic continuity and physiologic function, with the tongue being reduced in length and width.

\section{NASAL RECONSTRUCTION}

Procedures such as nasoseptoplasty, nasal turbinectomies, columella narrowing, enlargement of the luminal valves, nasal polypectomies, and reconstruction of external cartilage and bone may be indicated for correction of nasal airway obstruction. These are usually used as adjuncts in combination with other surgical procedures because their role in treating multilevel OSA is very limited.

\section{TONSILLECTOMY AND ADENOIDECTOMY}

Hypertrophied tonsillar and adenoid tissues can contribute to airway obstruction at the nasopharyngeal and oropharyngeal levels, especially in children and adolescents. Clinical and radiographic (lateral cephalometric x-ray) examination, as well as nasopharyngoscopy, can usually identify involvement of these structures. Tonsillectomy and adenoidectomy can be performed to eliminate the obstructions.

\section{MAXILLOMANDIBULAR ADVANCEMENT SURGERY (ORTHOGNATHIC SURGERY)}

Over the past few years, combined advancement of the maxilla and mandible has become the surgical procedure of choice for treatment of OSA in patients with decreased oropharyngeal airways, and numerous studies have reported the beneficial effects on the PAS (13-17).

Studies have shown that patients with a diminished crosssectional area of the pharynx may be predisposed to pharyngeal collapse and OSA $(18,19)$. It has been clearly documented that maxillomandibular advancement surgery is very efficacious in eliminating OSA by enlarging the PAS and tightening the upper airway muscles and tendons (velopharyngeal and suprahyoid muscles) by advancement of their bony origin (15-20).

Prevalence of OSA has also been linked to specific facial morphology types. Pracharktam et al found that variables related to soft tissues, hyoid bone to mandibular plane, body mass index, and soft palate length had the highest predictive value (18). Riley et al reported that a PAS of $<11 \mathrm{~mm}$ and a mandibular planehyoid bone angle $>15.4^{\circ}$ were indicative of OSA (21). Maxillomandibular advancement surgery has the benefits of correcting the patient's facial and occlusal deformities while also addressing OSA symptoms very effectively by enlarging the size of the oral cavity and pulling the base of the tongue and soft palate forward, thus increasing the PAS.

\section{PRESURGICAL CONSIDERATIONS}

Planning the skeletal, soft tissue, and dental correction of craniofacial deformities requires a comprehensive collection and analysis of data from various sources, including the patient's description of the problem and medical and dental history, clinical evaluation, radiographic examination, and dental model analysis. Orthodontic and dental treatment may be required before and/or after surgery to maximize the functional and aesthetic results of the orthognathic surgical procedures performed.

Careful attention is paid to the evaluation of the facial form in all 3 dimensions. Besides the clinical examination, the lateral cephalometric radiograph is of particular value, as it gives information about the facial skeletal and soft tissue structures as well as the PAS (normal dimension is $11 \pm 2 \mathrm{~mm}$ ) (16). The PAS in OSA patients is often narrower than in normal controls (22-24). Although PAS evaluation on a lateral cephalometric radiograph represents only a 2-dimensional overview of a 3-dimensional problem and is not taken in a supine sleeping position, when properly taken it provides very useful information in evaluating the anatomic interrelationships of the airway structures and in estimating tongue and nasopharyngeal volume.

Bony and soft tissue landmarks on the lateral cephalometric radiograph are analyzed with specific angular and linear measurements to aid in diagnosis and treatment planning. Surgical treatment objective tracings are constructed to predict the surgical movements required to correct the OSA and any coexisting associated facial deformity. The surgery is then simulated on dental plaster models of the patient's teeth and jaw structures mounted on a jaw articulator. Acrylic surgical stabilizing splints are fabricated in the laboratory on these plaster models, which reflect the jaws in their new position. These surgical splints help in the accurate repositioning of the jaws during surgery.

\section{SURGICAL PROCEDURES}

The most common orthognathic surgical procedures used in OSA correction are the maxillary Le Fort I osteotomy and the bilateral mandibular ramus sagittal split osteotomy. Using both maxillary and mandibular osteotomies usually allows for greater advancement of the jaw structures in OSA treatment, providing a better outcome. Wolford et al were the first to show that orthognathic surgery with counterclockwise advancement of the maxilla and mandible maximizes the increase of the PAS while also optimizing facial aesthetics $(25,26)$. Adjunctive procedures to correct airway obstruction in other areas (e.g., septoplasty, turbinectomies, external nasal reconstruction, modified UPPP, reduction glossectomy, tonsillectomy, adenoidectomy) can be performed concurrently with the jaw surgery. Bone plates and screws are used to rigidly stabilize the jaw structures during surgery. This eliminates the need for wiring the jaws together after 
surgery, thereby increasing patient comfort, permitting immediate active jaw function, improving dietary intake and oral hygiene, allowing normal speech, and minimizing chances of airway compromise immediately after surgery.

\section{CLINICAL AND RESEARCH EXPERIENCE}

The senior author of this paper (Dr. Wolford) has $>20$ years of experience in using orthognathic surgery for treatment of OSA. We have observed that many patients with OSA symptoms have common clinical and radiographic characteristics, including retruded mandible, retruded maxilla, posterior vertical maxillary deficiency, retropositioned tongue, high occlusal plane and high mandibular plane angulations, short chin-neck line, and decreased PAS on lateral cephalogram (25). Other characteristics that may be present include nasal airway obstruction (e.g., narrow nostrils, wide columella, enlarged turbinates, deviated septum, polyps, nasopharyngeal adenoid tissue, decreased posterior choanal height, constricted luminal valves, external nasal deformity) and oropharyngeal abnormalities (e.g., elongated soft palate and uvula, medially and posteriorly positioned posterior faucial pillars, enlarged adenoids, hyperplastic tonsils, macroglossia). Clinically, the tongue may appear large and retropositioned due to the decreased oral cavity volume resulting from the retruded position of the jaws.

\section{Patients and methods}

We performed a retrospective study to evaluate the effects of orthognathic surgery on the PAS anatomy. The treatment records of 72 patients (14 men, 58 women) operated on by Dr. Wolford for correction of dentofacial deformities (not all with OSA) at Baylor University Medical Center were retrospectively analyzed. All patients underwent Le Fort I maxillary osteotomies and mandibular ramus sagittal split osteotomies with rigid fixation for counterclockwise advancement of the maxillomandibular complex. Although many of these patients had turbinectomies and nasoseptoplasties at surgery, none had bony genioplasties, UPPP, adenoidectomies, tonsillectomies, or reduction glossectomies. Standardized lateral cephalometric radiographs were taken before surgery (T1) and at longest follow-up (T2) intervals. T1 and T2 radiographs were traced on acetate sheets and superimposed by an examiner to assess changes in pharyngeal anatomy. The PAS dimensions were calculated by measuring the narrowest dimension from the posterior pharyngeal wall to the tongue base and to the soft palate. A Student $t$ test was used to assess the statistical significance of results. A $P$ value $<0.0001$ was considered statistically significant.

\section{Results}

The average age of the patient sample was 36.3 years (range, 12 to 56 years), and the average follow-up time was 3 years (range, 1 to 8.1 years). The mean mandibular advancement was $12 \mathrm{~mm}$ (SD, 5.4) measured at the genial tubercles. The mean maxillary advancement at point $A$ was $5.1 \mathrm{~mm}(\mathrm{SD}, 2.1)$. At the soft palate, the mean PAS presurgery was $7.9 \mathrm{~mm}(\mathrm{SD}, 2.8)$ and postsurgery was $14.1 \mathrm{~mm}(\mathrm{SD}, 3.7)$, for a mean increase of 6.2 $\mathrm{mm}(P<0.0001 ; \mathrm{SD}, 3.1)$. At the tongue base, the mean PAS presurgery was $7.7 \mathrm{~mm}(\mathrm{SD}, 2.6)$ and postsurgery was $15.8 \mathrm{~mm}$ $(\mathrm{SD}, 3.6)$, for a mean increase of $8.1 \mathrm{~mm}(P<0.0001$; SD, 3.6).
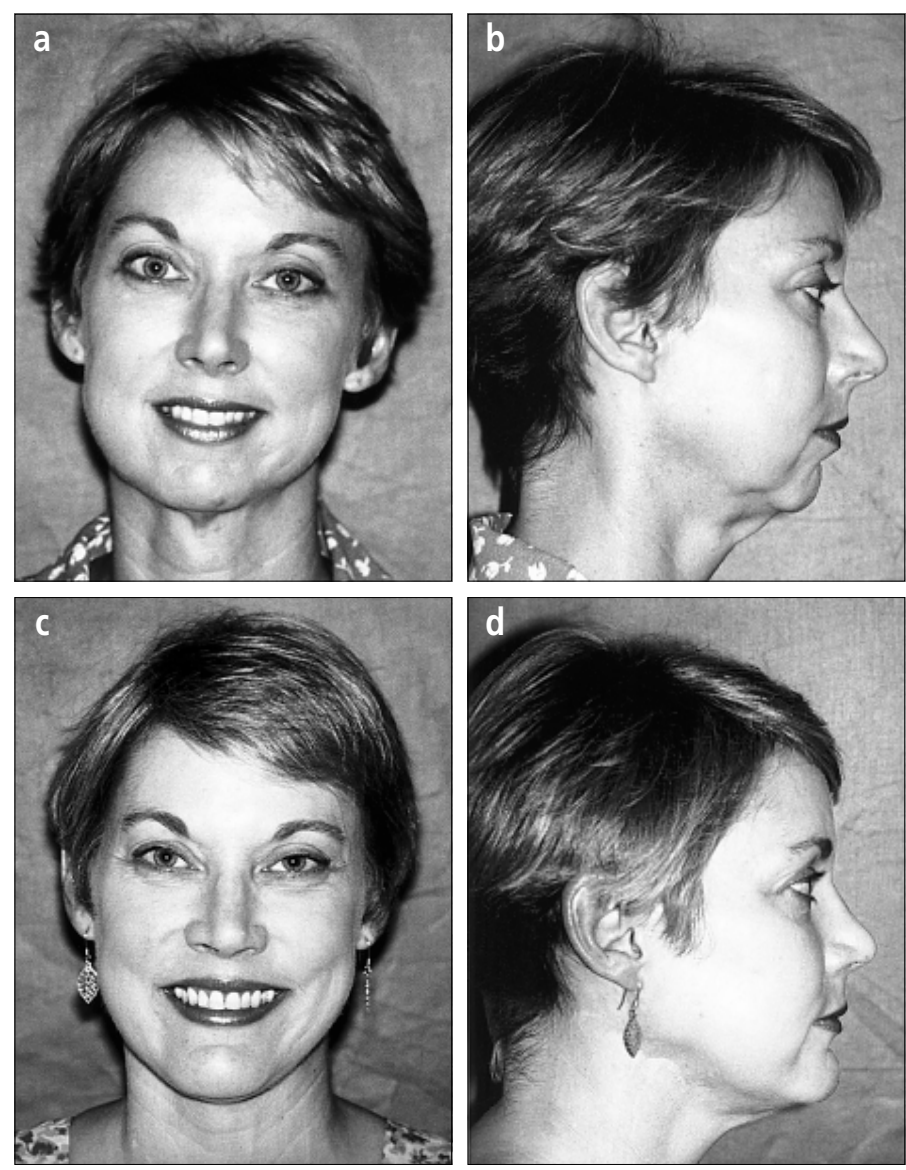

Figure 5. (a) Frontal view of a 49-year-old woman who was referred by her physician for correction of OSA symptoms. (b) Profile view shows the presence of a dentofacial deformity with retruded upper and lower jaws. (c) The patient 5 years after maxilla and mandible advancement and intranasal surgery. The sleep apnea symptoms have been eliminated. (d) Profile view shows a good aesthetic result with harmonious facial balance.

Thus, the mean PAS increase ranged from $52 \%$ to $63 \%$ of the amount of mandibular advancement performed. No patients had any significant OSA symptoms at longest follow-up.

\section{CASE PRESENTATION}

A 49-year-old woman (Figure 5a, 5b) was referred for treatment of OSA symptoms by her physician. She gave a history of loud snoring while sleeping, daytime somnolence, generalized fatigue, and nasal airway obstruction. She had been using a CPAP machine while sleeping for 1 year prior to presentation, without significant relief of symptoms. Other previously failed nonsurgical OSA therapy included weight loss, use of medications, and orthodontic/dental appliance therapy. A presurgical polysomnographic study showed severe sleep apnea with a respiratory disturbance index of 51 and a mean oxygen saturation of $84 \%$.

Presurgical clinical and radiographic evaluation (Figure 6a) revealed a diagnosis of 1) mandibular retrusion; 2) maxillary retrusion; 3) posterior vertical maxillary deficiency; 4) severely decreased PAS (2 $\mathrm{mm})$; 5) high occlusal and mandibular plane angles; 6) deviated nasal septum; 7) hypertrophied nasal turbinates; 8) wide nasal columella, creating narrow nostrils; and 9) nasal airway obstruction caused by the 3 previous diagnoses.

After the necessary orthodontic treatment was completed, the following surgical procedures were performed at 1 operation 


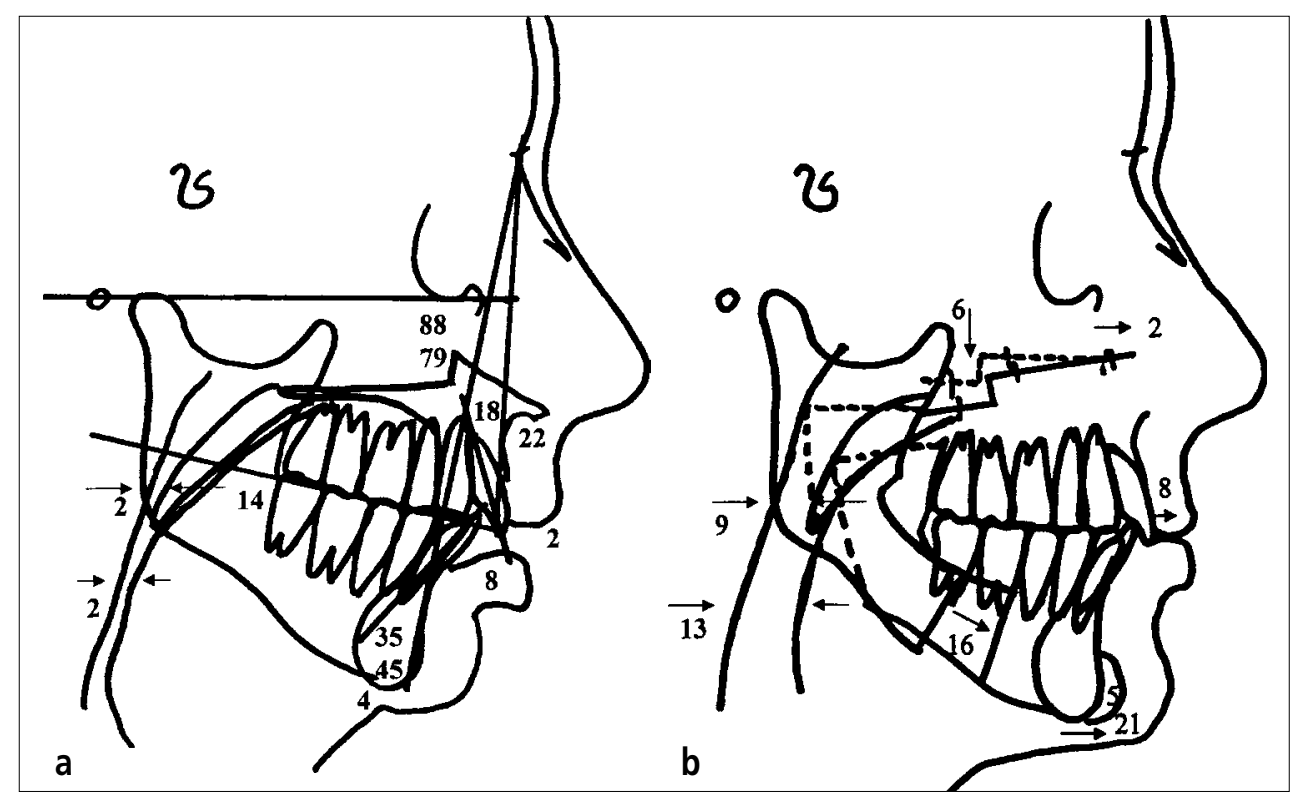

Figure 6. (a) Presurgical tracing of the dentofacial structures on the lateral cephalometric x-ray. Note the narrowed PAS of $2 \mathrm{~mm}$ (normal is $11 \pm 2 \mathrm{~mm}$ ). (b) Postsurgical tracing of the dentofacial structures on the lateral cephalometric $x$-ray showing the effects of surgery performed on the upper and lower jaws. Note the increased dimension of the PAS to $13 \mathrm{~mm}$ at the tongue base and $9 \mathrm{~mm}$ at the soft palate.

(Figure 6): 1) multiple-segment Le Fort I osteotomies to advance the upper jaw $8 \mathrm{~mm}, 2$ ) bilateral mandibular ramus sagittal split osteotomies to advance the lower jaw $16 \mathrm{~mm}, 3$ ) a 5 -mm chin implant, 4) a nasal septoplasty; 5) bilateral partial inferior turbinectomies, and 6) nasal columellar narrowing to enlarge the nostrils.

The chin point advanced $21 \mathrm{~mm}$ forward from its presurgical position (as a result of the mandibular advancement and chin implant). The PAS at the tongue base increased to $13 \mathrm{~mm}(650 \%$ improvement from the presurgery PAS of $2 \mathrm{~mm}$ ) and at the soft palate level increased to $9 \mathrm{~mm}$ (450\% improvement from the presurgery PAS of $2 \mathrm{~mm}$ ) (Figure 6b). Postoperative polysomnography revealed a significant improvement, with a respiratory disturbance index of 5 (presurgery was 51) and a mean oxygen saturation of $95 \%$ (presurgery was $84 \%$ ). Five years after surgery, the patient had a stable clinical result (Figure $5 c, 5 d$ ). She does not suffer from snoring, daytime somnolence, or fatigue and has not required the use of CPAP since surgery.

1. Thornton WK, Roberts DH. Nonsurgical management of the obstructive sleep apnea patient. J Oral Maxillofac Surg 1996;54:1103-1108.

2. Weigland L, Zwillich CW. Obstructive sleep apnea. Dis Mon 1994:40:197252.

3. Kuhlo W, Doll E, Frank MD. Erfolgrieche Behandlung eines PickwickSyndroms durch eine Dauertrachealkanuele. Dtsc Med Wochenschr 1969; 94:1286-1290.

4. Tiner BD. Surgical management of obstructive sleep apnea. J Oral Maxillofac Surg 1996;54:1109-1114.

5. Riley RW, Guilleminault C, Powell NB, Derman S. Mandibular osteotomy and hyoid bone advancement for obstructive sleep apnea: a case report. Sleep 1984;7:79-82.

6. Ikematsu T. Study of snoring, 4th report: therapy. Journal of Japanese Otorhinolaryngology 1964;64:434-435.

7. Fujita S, Conway W, Zorick F, Roth T. Surgical correction of anatomic abnormalities in obstructive sleep apnea syndrome: uvulopalatopharyngo- plasty. Otolaryngol Head Neck Surg 1981; 89:923-934.

8. Simmons FB, Guilleminault C, Silvestri R. Snoring, and some obstructive sleep apnea, can be cured by oropharyngeal surgery. Arch Otolaryngol 1983;109:503-507.

9. Simmons FB, Guilleminault C, Miles LE. The palatopharyngoplasty operation for snoring and sleep apnea: an interim report. Otolaryngol Head Neck Surg 1984;92:375_ 380.

10. Katsantonis GP, Schweitzer PK, Branham GH, Chambers G, Walsh JK. Management of obstructive sleep apnea: comparison of various treatment modalities. Laryngoscope 1988;98:304-309.

11. Guilleminault C, Hayes B, Smith L, Simmons FB. Palatopharyngoplasty and obstructive sleep apnea syndrome. Bull Eur Physiopathol Res 1983;19:595-599.

12. Ryan CF, Love LL. Unpredictable results after laser assisted uvuloplasty in the treatment of obstructive sleep apnea. Thorax 2000;55:399-404.

13. Wolford LM, Cottrell DA. Diagnosis of macroglossia and indications for reduction glossectomy. Am J Orthod Dentofacial Orthop 1996;110:170-177.

14. Guilleminault C, Hill MW, Simmons FB, Dement WC. Obstructive sleep apnea: electromyographic and fiberoptic studies. Exp Neurol 1978;62:4867.

15. Li KK, Riley RW, Powell NB, Troell R, Guilleminault C. Overview of phase II surgery for obstructive sleep apnea syndrome. Ear Nose Throat J 1999; 78:851-857.

16. Riley RW, Powell NB, Guilleminault C. Obstructive sleep apnea syndrome: a surgical protocol for dynamic airway reconstruction. J Oral Maxillofac Surg 1993;51:742-747.

17. Reiche-Fischel $O$, Wolford LM. Posterior airway space changes after double jaw surgery with counter-clockwise rotation. J Oral Maxillofac Surg 1996; 54:96.

18. Pracharktam N, Nelson S, Hans MG, Broadbent BH, Redline S, Rosenberg C, Strohl KP. Cephalometric assessment in obstructive sleep apnea. Am J Orthod Dentofacial Orthop 1996;109:410-419.

19. Riley RW, Guilleminault C, Herran J, Powell N. Cephalometric analyses and flow-volume loops in obstructive sleep apnea patients. Sleep 1983; 6:303-311.

20. Riley RW, Powell NB, Guilleminault C. Current surgical concepts for treating obstructive sleep apnea syndrome. J Oral Maxillofac Surg 1987;45:149_ 157.

21. Guilleminault C, Riley RW, Powell N. Obstructive sleep apnea and abnormal cephalometric measurements. Implications for treatment. Chest 1984; 86:793-794

22. Lowe AA, Gionhaku N, Takeuchi K. Three-dimensional CT reconstructions of tongue and airway in adult subjects with obstructive sleep apnea. Am J Orthop Dentofacial Orthop 1986;90:364-374.

23. Haponik EF, Smith PL, Bohlman ME, Allen RP, Goldman SM, Bleecker ER. Computerized tomography in obstructive sleep apnea. Correction of airway size with physiology during sleep and wakefulness. Am Rev Respir Dis 1983;127:221-226.

24. Suratt PM, Dee P, Atkinson RL, Armstrong P, Wilhort SC. Fluoroscopic and computed tomographic features of pharyngeal airway in obstructive sleep apnea. Am Rev Respir Dis 1983;127:487-492.

25. Wolford LM, Chemello PD, Hilliard FW. Occlusal plane alteration in orthognathic surgery-Part I: Effects on function and esthetics. Am J Orthod Dentofacial Orthop 1994;106:304-316.

26. Chemello PD, Wolford LM, Buschang MS. Occlusal plane alteration in orthognathic surgery-Part II: Long-term stability of results. Am J Orthod Dentofacial Orthop 1994;106:434-440. 